\title{
FERMILAB-SLIDES-18-795-E
}

\section{Design of the Mu2e Straw Tracker}

\section{Kate Ciampa}

University of Minnesota

\section{New Perspectives at Fermilab}

June 19, 2018

This document was prepared by Mu2e collaboration using the resources of the Fermi National Accelerator Laboratory (Fermilab), a U.S. Department of Energy, Office of Science, HEP User Facility. Fermilab is managed by Fermi Research Alliance, LLC (FRA), acting under Contract No. DE-ACo2-07CH11359. 


\section{Tracker in the Context of the Muze Apparatus}

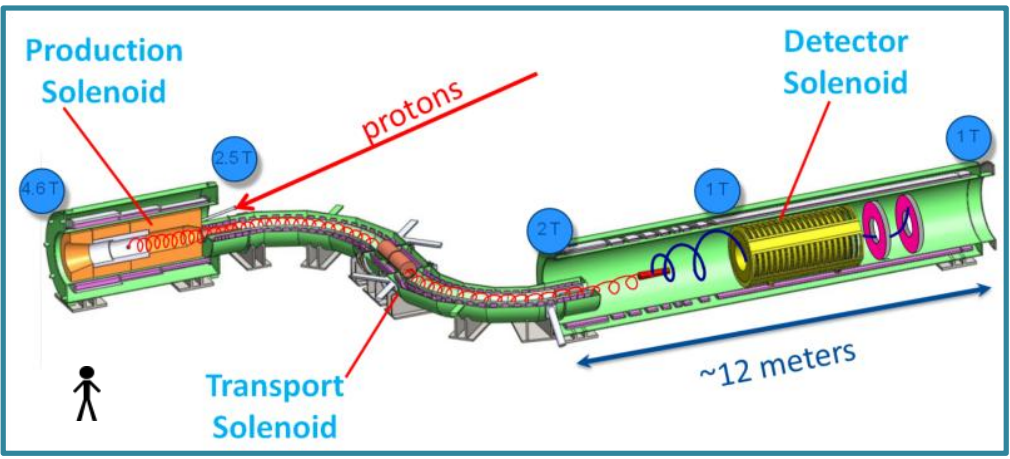

- The Mu2e experiment searches for charged lepton flavor violation, when a muon converts to an electron inside an aluminum atom

- In the Detector Solenoid, tracker and calorimeter measure properties of signal electrons from this process

- Electrons move downstream from stopping target, and tracker determines momenta by fitting their helical trajectories through a magnetic field 


\section{Mu2e Tracker Challenges}

Beam Enters

Mu2e Detector

Solenoid

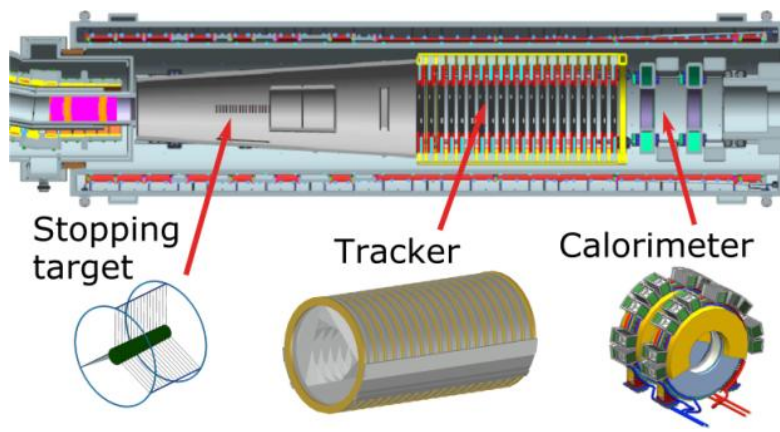

- Sensitivity goal for the muon conversion measurement sets challenging physics goals for the tracker

- Must determine electron momenta with better than $0.2 \%$ resolution

- Use minimal material to avoid signal electron energy loss

- Maintain efficiency at high hit rates

- Operate in a strong magnetic field and high radiation environment 


\section{Energy Loss in Material}

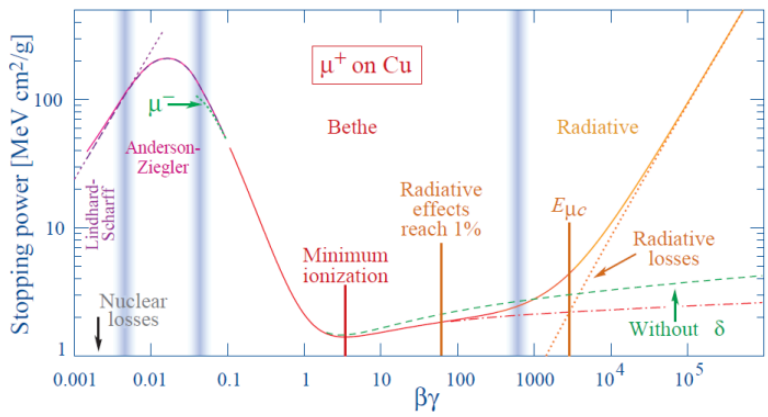

Example of energy loss for muon

For comparison, Mu2e electron has $\gamma B=205$

- Particles lose energy as they pass through material, and Mu2e must minimize this effect for signal electrons

- Energy as a function of distance traveled decreases more rapidly for material with more protons, characterized by radiation length $\mathrm{X}_{0}$

$$
E(x)=E_{0} \exp \left(\frac{-x}{X_{0}}\right) \text { with } \mathrm{X}_{0} \sim 8.9 \mathrm{~cm} \text { for aluminum }
$$

- The Mu2e tracker can only use a very small amount of metal - even just $3 \mathrm{~cm}$ of aluminum would cause $30 \%$ energy loss for electron 


\section{Signal Electron vs. Background Spectrum}

- Muon conversion produces a monoenergetic electron with kinetic energy nearly equal to the muon rest mass, $E_{e} \sim 105 \mathrm{MeV}$

- Energy from normal muon decay at rest is shared among electron and neutrinos, with maximum $E_{e}=m_{\mu} / 2$

- For muon orbiting a nucleus in the aluminum target, decay spectrum has high energy tail that extends out to signal region

- Tracker must measure momentum with better than $0.2 \%$ resolution to separate conversion electrons from normal muon decay products
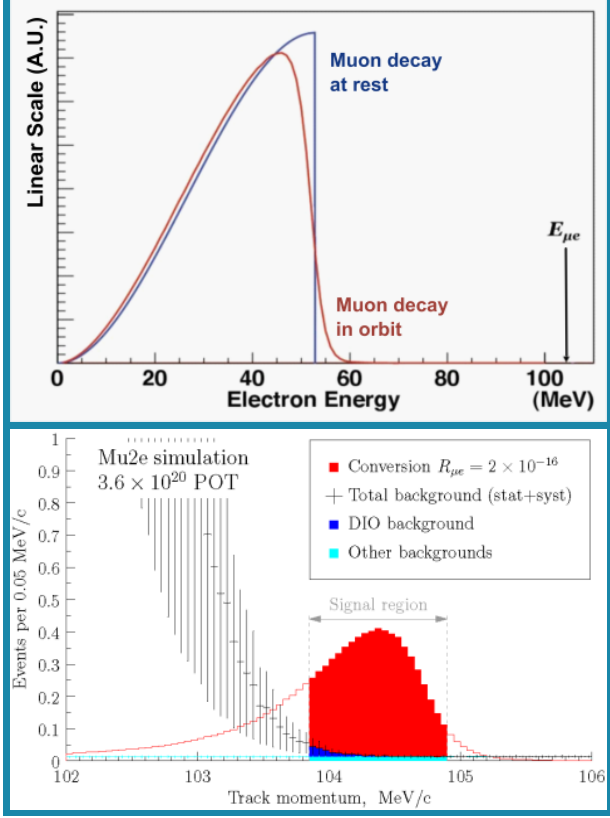
How is momentum measured with sufficient precision to identify conversion electron candidates? 
- The tracker will use over 20,000 straw drift cells to locate high energy electrons based on ionization events along their trajectories

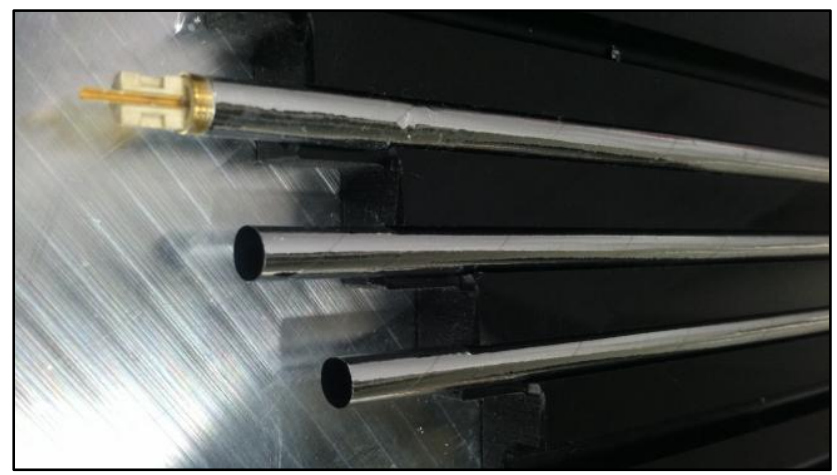

Mu2e Straws 


\section{Mu2e Tracker Components - Panels}

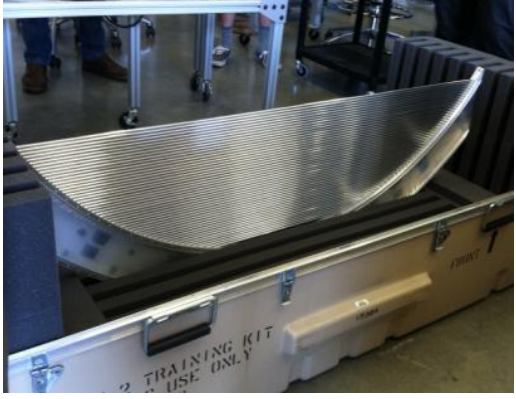

Mu2e Panel
- A panel holds 96 straws parallel under tension, and has space for readout electronics

- Panels of straws arranged in a circle around the beamline intercept only electrons with large transverse momentum

- Low energy electrons from muon decay, and beam muons, will pass through the hole along the axis of the detector

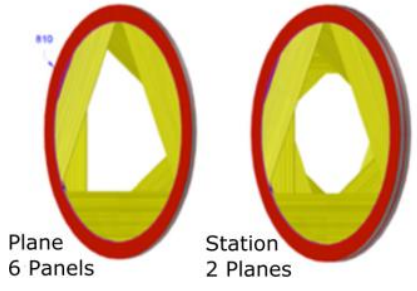

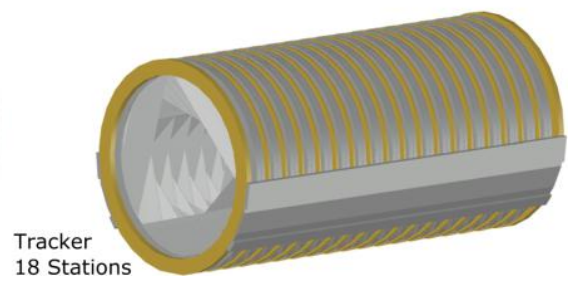




\section{A Mass Spectrometer for a Massive Quantity of Electrons}

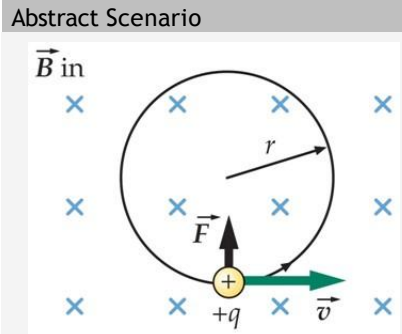

- A particle of mass $m$ and charge $q$ moves perpendicular to a magnetic field. What is the radius of its trajectory?

- Magnetic force provides centripetal acceleration

$$
F=|q \vec{v} \times \vec{B}|=\frac{m v^{2}}{r}
$$

- Track radius found from known momentum, or vice versa

$$
r=\frac{m v_{\perp}}{q B}=\frac{p_{\perp}}{q B}
$$

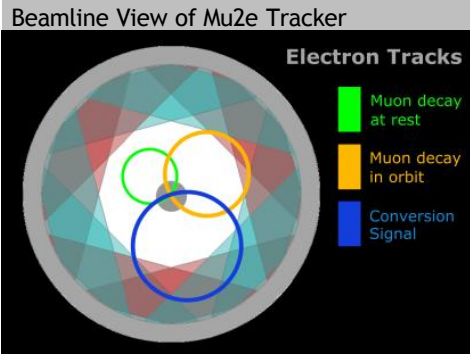

- In Mu2e detector, magnetic field is uniform 1T and signal electron has $\mathrm{q}=-\mathrm{e}$

- For limiting case where all momentum is transverse, $\mathrm{p}_{\perp}=105 \mathrm{MeV} / \mathrm{c}$

$$
r=\frac{p_{\perp}}{e B}=35 \mathrm{~cm}
$$

Mu2e tracker instrumentation is positioned to contain these trajectories 
How is a conversion electron trajectory located within the tracker? 
- The tracker will use over 20,000 straw drift cells to locate high energy electrons based on ionization events along their trajectories

- To minimize multiple scattering, which alters electron momentum, straws use minimal material and are surrounded by vacuum

- Straws account for only about half of the active detector mass, comparable to the drift gas inside

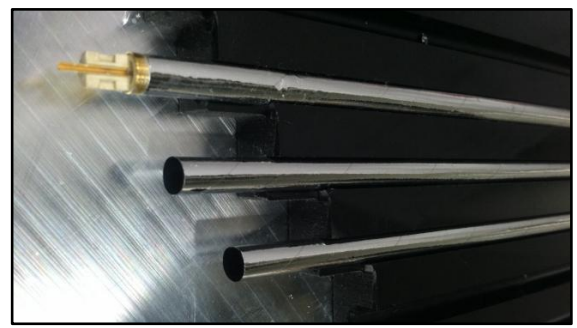

Mu2e Straws 
- Straws are made of low-mass metalized Mylar

- Wound from $15 \mu \mathrm{m}$ thick strips, with layers of aluminum to separate drift gas from vacuum and gold for conductivity

- Similar in diameter to drinking straws, with lengths varying from 44 to $114 \mathrm{~cm}$
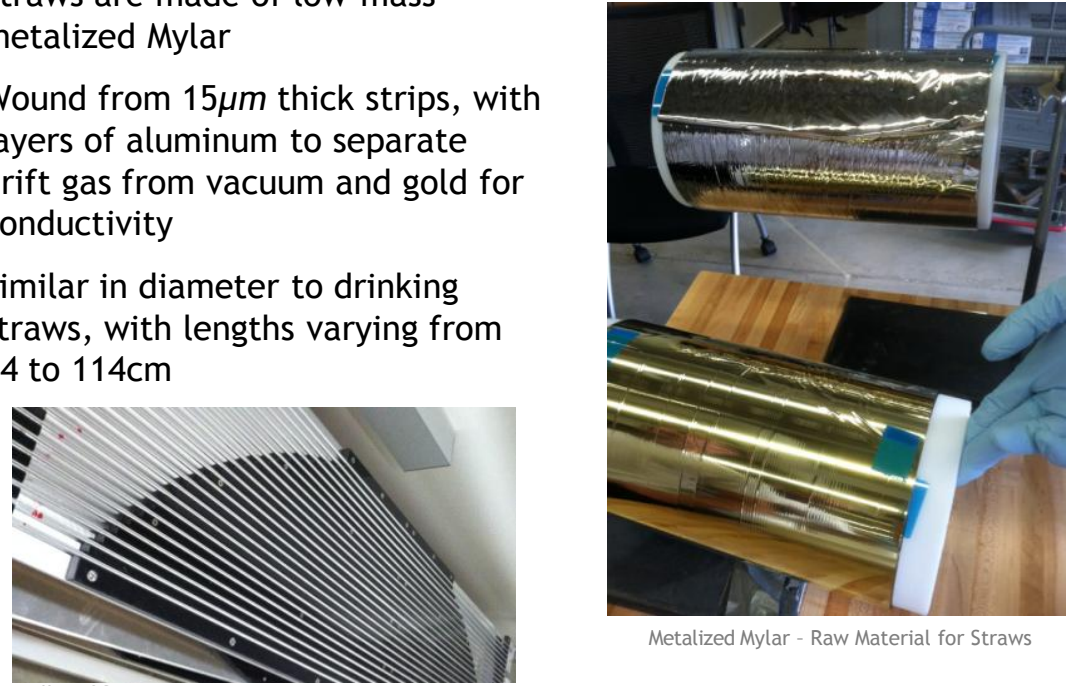

Metalized Mylar - Raw Material for Straws 


\section{Signal Electron Path Through Straws}

- A high energy electron ionizes $\mathrm{Ar}: \mathrm{CO}_{2}$ gas in the straws, producing a cascade of secondary electrons that drift toward a central $25 \mu \mathrm{m}$ sense wire

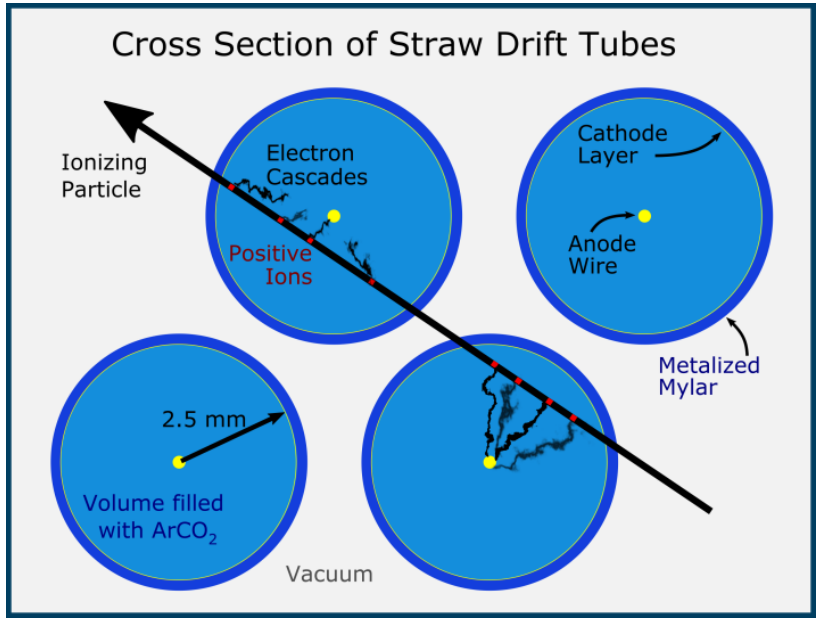




\section{Intro Physics in Real Life: Gauss' Law}

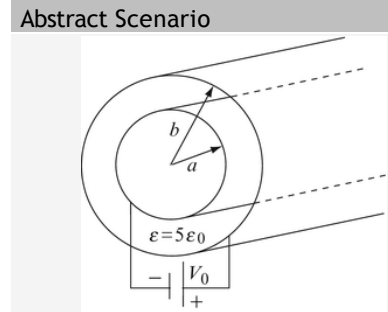

- Electric field between conductors with cylindrical symmetry can be found with Gauss' Law

$$
\oint \vec{E} \cdot d \vec{A}=\frac{Q_{e n c}}{\varepsilon}
$$

- Effective charge $\lambda$ found by relating field to potential difference

$$
\Delta V=-\int \vec{E} \cdot d \vec{l}=\frac{-\lambda}{2 \pi \varepsilon} \ln (r)+\text { const } .
$$

\section{Mu2e Straw and Sense Wire}

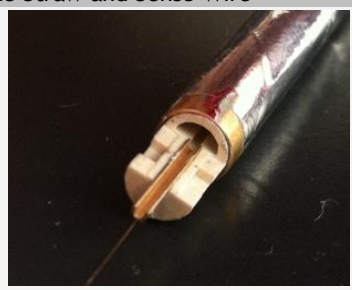

- Straw is grounded (cathode) with radius $r_{s}=2.5 \mathrm{~mm}$, and sense wire is at $1450 \mathrm{~V}$ (anode) with radius $r_{w}=12.5 \mu \mathrm{m}$

$$
E(r)=\frac{V_{w}}{r \ln \left(r_{s} / r_{w}\right)}
$$

nue to this electric field, secondary electrons drift toward sense wire

- Displacement of wire inside straw, or increase in voltage, can cause electrical breakdown 
When an ionization event takes place in a straw, how is it detected? 
- Just outside active detector region, front-end electronics will amplify and digitize the signal from ionization events

- Highly segmented detector capable of readout efficiency in high hit rate averaging around $15 \mathrm{kHz} / \mathrm{cm}^{2}$

- Electronics must also withstand neutron and photon flux produced when muons interact with stopping target nuclei

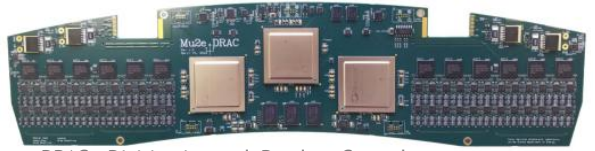

DRAC - Digitization and Readout Control
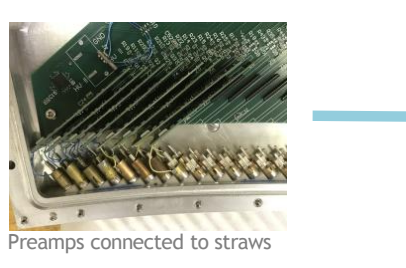


\section{Ionization Signal}

- Readout at both ends of the straw constrains hit position along straw axis, within several $\mathrm{cm}$

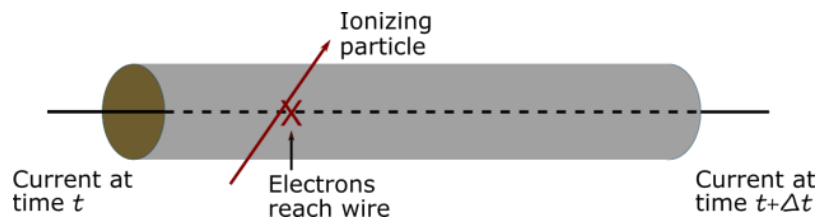

- Timing of the current pulse from an electron cascade gives the distance from ionization event to wire, based on electron drift velocity

- Measure current pulse time to within 2 ns for $100 \mu m$ resolution in hit position 
With so many particles passing through the tracker, how do we know if a hit is from a conversion electron or another particle? 


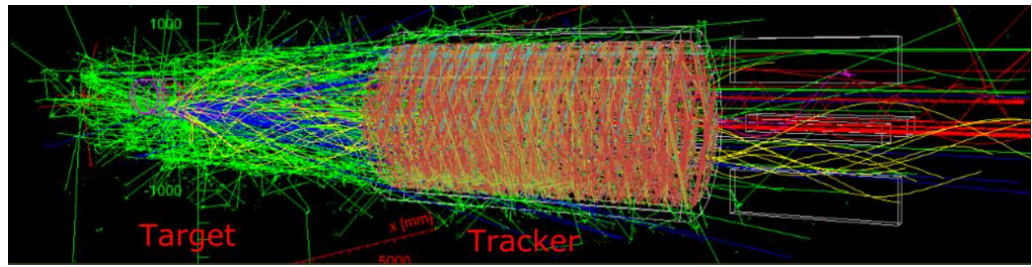

- Particle trajectories are simulated segment by segment using Monte Carlo methods in GEANT4, based on measured properties of the tracker

- Analysis goal: distinguish pattern of conversion electron hits

- Group hits by time and fit geometrically, using several iterations to reach a helix with $<0.2 \%$ momentum resolution

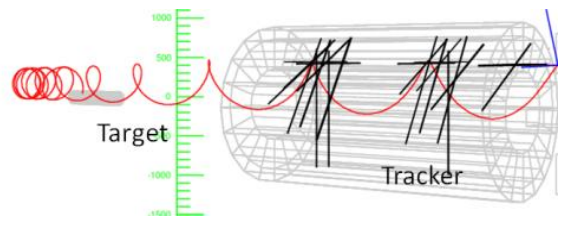




\section{Exciting Times Ahead...}

- Muze has the potential to discover charged lepton flavor violation in muon to electron conversion, a previously unobserved process that would indicate the existence of new physics

- To reach a single event sensitivity of $3 \times 10^{-17}$, electron momenta must determined with better than $0.2 \%$ resolution

- The Mu2e solution to this challenge is an innovative straw tracker design

- Drift cell straws have ultra low mass to minimize energy loss and multiple scattering

- A central hole to avoid beam particles and low energy electrons

- Highly segmented active detector volume to maintain efficiency at high hit rates

...check back in 2021 to see the initial physics data! 